\section{Deficiencias en los diagnósticos de las reformas sanitarias de los años noventa en América Latina}

\author{
María del Pilar Guzmán Urrea
}

Forma de citar: Guzmán Urrea MP. Deficiencias en los diagnósticos de las reformas sanitarias de los años noventa en América Latina. Rev Panam Salud Publica 2009;25(1):84-92.

Palabras clave: sistemas de salud, salud pública, administración y planificación en salud, equidad en salud, América Latina.

1 Universidad El Bosque-Humanidades, Bogotá, Colombia. La correspondencia deberá enviarse a la profesora Ma. del Pilar Guzmán Urrea, Universidad El Bosque-Humanidades, Calle 132 No. $7^{\text {a }} 35$ Bogotá, Colombia. T.: 571-2588148. Correo electrónico: mapiguzman@ tutopia.com
Al final de los años ochenta y principios de los noventa, en la mayoría de los países occidentales se generalizó un movimiento de reformas sanitarias que autores como Almeida (1) ubican en la tercera generación de reformas en el sector salud del siglo $\mathrm{XX}^{2}$, estrechamente relacionado con la "agenda post-welfare" y la tendencia internacional de reforma del Estado que puso sobre la mesa de discusión temas como el papel del Estado y del mercado en el sector sanitario, la descentralización, la privatización y la concesión de mayor capacidad de elección a los "consumidores" de los servicios de salud.

Si bien cabe reconocer que existe una amplia variedad de estrategias en las agendas de cambio en los diferentes países, determinadas por aspectos históricos, socioeconómicos, culturales, de contexto, de proyectos políticos, etc., que derivan en innumerables especificidades en cada país, es posible distinguir un conjunto de tendencias paralelas tales como:

1. La racionalización de los recursos sanitarios a través del establecimiento de prioridades, el aumento de la coparticipación financiera de los usuarios, la búsqueda de una producción sanitaria más eficiente y la distribución de los recursos de manera efectiva.

2. La incorporación del concepto de "competencia administrada" como forma de establecer mercados "regulados" en el ámbito sanitario y de generar medidas de control que operen sobre la oferta y la demanda de servicios de salud.

3. La separación entre el financiamiento y la provisión de servicios cuyo objetivo apunta a disminuir la producción directa de servicios por parte del Estado, a cambio de fortalecer sus funciones de regulación.

4. La descentralización de las responsabilidades en el financiamiento y la prestación de los servicios tanto en los planos subnacionales de gobierno como para el sector privado (2)

Los efectos de estas propuestas de cambio dependen del país en el que se estén aplicando; así por ejemplo, en los países de Europa Occidental que poseen sistemas de salud públicos, si bien se han llevado a cabo reformas encaminadas a la separación de las

\footnotetext{
2 La primera generación de reformas corresponde al período comprendido entre finales del siglo XIX y la I Guerra Mundial, cuando se crearon los programas específicos para pobres y trabajadores de baja renta y los programas nacionales de salud. La segunda generación de reformas se dio después de la II Guerra Mundial con la consolidación y universalización de los servicios de salud, la creación de sistemas nacionales de salud y la declaración del derecho a la salud como derecho de ciudadanía.
} 
funciones de financiamiento y provisión, la flexibilización de la gestión y la transformación en la asignación de los recursos sanitarios, siguen siendo muy valorados por sus ciudadanos los principios de universalidad, solidaridad e inclusión y la atención sanitaria se sigue considerando como un derecho de ciudadanía o un derecho social (3). Por el contrario, en el caso de América Latina —-donde los niveles de exclusión y marginalidad social son tan altos y la intervención del Estado en la salud tradicionalmente ha sido bastante fragmentada (dedicada a grupos específicos de la población), con escasa capacidad de regulación de la prestación privada y donde la hegemonía de la doctrina neoliberal se ha aplicado de manera rigurosa (debido en buena parte a la fuerte influencia de organismos financieros internacionales como el Banco Mundial y el Fondo Monetario Internacional)_, los efectos de esas estrategias han ahondado aún más las inequidades de los sistemas de salud de la región, debilitado sus ya frágiles y parciales estructuras de derechos de ciudadanía y han revitalizado la participación privada en el financiamiento y la provisión sanitaria (2).

La reflexión que se pretende realizar en este artículo no está orientada a una revisión detallada de los aspectos "contextuales" de las diferentes reformas sanitarias implementadas a lo largo de los años noventa que, como se anotaba anteriormente, determinan el curso particular de las reformas en cada país, sino al análisis de algunas de las premisas que son comunes en dichas reformas. Para su desarrollo se recurrió a la revisión de fuentes secundarias relacionadas con temas de salud pública, salud colectiva y de justicia sanitaria.

El trabajo se divide en cuatro partes: la primera, analiza la visión "tecnocrática" que ha predominado en los diagnósticos de las reformas, la cual sobrevalora el desempeño de las instituciones de salud en el estado de salud de la población, limitando el objetivo de las reformas a la búsqueda de la eficiencia de las instituciones y la reducción del gasto en este sector. En estrecha relación con este aspecto, en la segunda parte se cuestiona la forma como la visión económico-gerencial de las reformas reduce toda la complejidad del proceso salud-enfermedad al ámbito curativo, especialmente al hospital como centro de los cambios en salud y no tiene en cuenta los enfoques que plantean los determinantes sociales de dicho proceso y la necesidad de formular estrategias "intersectoriales" en las reformas. En la tercera parte se revisa uno de los aspectos más polémicos en las reformas sanitarias de los años noventa: ¿cómo armonizar las metas de la equidad y la eficiencia en las políticas de salud? Este tema es extenso, en tanto que conceptos como el de la "equidad" se adaptan a diferentes proyectos políticos y tendencias ideológicas. Lo que se intenta mostrar en este trabajo principalmente, es la dificultad que supone considerar la equidad en el consumo de los servicios de salud como condición "suficiente" para disminuir las desigualdades en la salud de la población. De igual forma, se muestran los efectos que algunas de las reformas han tenido sobre la equidad en salud, especial- mente en países como Colombia donde más se ha profundizado la introducción de los mecanismos de mercado en su sistemas sanitario. En la cuarta parte se estudia el efecto que provoca el aumento de las consideraciones con respecto a los factores de riesgo individual o de responsabilidad individual en el origen de las enfermedades, algunas de ellas expresadas de manera implícita en muchas de las estrategias de reformas sanitarias de los años noventa. A continuación se abordarán dichos aspectos.

\section{La creencia de que hacer más eficiente el funcionamiento de los sistemas de salud per se producirá una mejora en la salud de la población}

Una de las premisas que orientan las reformas sanitarias de los años noventa en América Latina es la confianza en que hacer más eficiente el funcionamiento de los sistemas de salud -especialmente lo relacionado con lo hospitalario- per se mejorará la salud de la población. Ello se refleja perfectamente en el afán de renovar las estructuras organizativas y financieras de los hospitales, sin que se haya hecho un replanteamiento de los fines prioritarios de los sistemas de salud.

El papel tan importante que se le sigue concediendo a los servicios de salud en el mejoramiento de la salud de la población desconoce o minimiza los factores socioeconómicos, políticos y culturales como determinantes en la etiología de las enfermedades, a pesar de que desde la década de 1970 múltiples investigaciones (4-6) vienen demostrando que el verdadero impacto en el control de enfermedades infecciosas de muchos de los avances médicos de la primera mitad del siglo XX (la aparición de los antibióticos, las quimioterapias y los programas de inmunización) fue mucho menor de lo que generalmente se suponía, pues factores como la mejora de las condiciones socio-económicas y una dieta más completa fueron fundamentales en el descenso sustancial y prolongado de la mortalidad. De igual manera, teorías como la planteada por Lalonde (7) y de Blum (8) acerca de las cuatro categorías de factores que inciden en las enfermedades, a saber: ambiente, comportamiento, herencia y servicios de salud, y los planteamientos realizados en la Conferencia Internacional sobre Atención Primaria en Salud de Alma Ata en 1978 (9), han mostrado las importantes conexiones entre el nivel de vida (alimentación, educación, suministro suficiente de agua potable y saneamiento básico) y el estado de salud de la población.

Además, con la promulgación de la Carta de Ottawa de 1986 se plantea la promoción de la salud como una corriente innovadora en tanto la salud se articula a las nociones de bienestar y calidad de vida y se definen estrategias para actuar sobre los determinantes de la salud, combinar métodos diversos y complementarios para incidir tanto en los individuos como en las políticas públicas y buscar la participación efectiva de la población (10). En este mismo sentido, trabajos más recientes como el de Evans y cols. (11) han presentado importantes evidencias de la existencia de una 
fuerte relación causal entre el estatus de salud, el estatus social y el ambiente físico, para demostrar que los factores que determinan la salud van más allá de la responsabilidad de los servicios de atención sanitaria.

A pesar de los aportes de dichos trabajos y de las declaraciones internacionales, en la mayoría de las reformas de salud propuestas en los años noventa se puede ver que, aunque se le ha otorgado un importante papel al médico general o al médico de familia, que podría sugerir un cierto cambio de rumbo en la orientación de las políticas hacia la prevención y promoción de la salud, dicho papel está concebido como parte de una estrategia para disminuir costos en la prestación asistencial (en tanto se convierte en un "filtro" en el acceso a los servicios de salud), pero no constituye un elemento central en la reflexión de la estructura sanitaria y sus fines, ni de los modelos de educación médica existentes.

Por otro lado, es importante tener en cuenta que el interés por los aspectos institucionales, organizativos y financieros en las reformas del sector salud se encuentra estrechamente relacionado con la consolidación de las propuestas de reforma del Estado difundidas a través del modelo neoliberal (adoptado en América Latina desde finales de los años ochenta y vinculado a los procesos de ajuste macroeconómico) expresadas, como lo anotan Gamboa y Arellano (12), en estrategias como la descentralización, la privatización, la búsqueda de flexibilidad interna de las organizaciones públicas y de cambios en su control, la promoción de la gestión laboral flexible y meritocrática, la organización centrada en el cliente, el financiamiento a la demanda en vez de financiar la oferta de servicios, la modernización de la información y la búsqueda de cambios en la cultura organizacional y ciudadana.

No todas estas propuestas se han impuesto con igual fuerza en la Región y sus consecuencias han sido heterogéneas, como se ha estudiado en diversos trabajos $(2,12-17)$. Algunas de las propuestas que más han fortalecido la concepción de la transformación gerencial de los servicios asistenciales de salud como la mejor vía para mejorar la salud de la población, son:

a) El gran interés en que las reformas del sector salud aumenten la capacidad de elección de los "clientes" o usuarios, con el fin de estimular la competencia, la eficacia y la calidad de los entes que prestan el servicio y el uso de herramientas de "marketing" para promocionar los servicios (básicamente los hospitalarios y muy poco los dirigidos a la prevención y promoción de la salud). Evidentemente, para el paciente la posibilidad de elección de las entidades aseguradoras y proveedoras es importante, pues puede facilitarle el ejercicio de su autonomía, en el sentido de poder hacer una escogencia libre y personal sobre la base de una información clara, completa y neutral en aspectos relativos a su diagnóstico y tratamiento. Sin embargo, la concesión de un mayor poder a los pacientes está centrada principalmente en los aspectos logísticos de la atención sanitaria (elección de hospitales y de profesionales) y está concebida como un ejercicio de derechos comerciales (contratos entre compradores y ven- dedores), con un enfoque "individualista" en el que cada uno se preocupa por acrecentar su propia capacidad de elección sin contemplar la de otros y en el que la "orientación al cliente" solo parece ser importante en tanto que esta resulta fundamental para aumentar la cuota de mercado.

Esta concepción de los pacientes como "consumidores" inaugura, además, el "paternalismo administrativo", en el cual son los gerentes, los administradores y los planificadores quienes, ante las fuertes presiones para disminuir los costos y aumentar la eficiencia de los sistemas sanitarios, pasan a ser considerados como los "expertos" con capacidad de decidir, incluso por encima de la autodeterminación de los médicos y del principio de autonomía de los pacientes. No se tiene en cuenta que la determinación de un verdadero control y poder de los pacientes va más allá de su intervención en el consumo o adquisición de un servicio sanitario e involucra su participación en aspectos esenciales como los procesos de establecimiento de prioridades, la asignación y distribución de los recursos sanitarios y las metas y fines que la sociedad espera que guíen a sus organizaciones sanitarias.

b) Desde el enfoque neoliberal se considera que las acciones relacionadas con la prevención de las enfermedades (control de calidad del aire, del agua, inmunizaciones, etc.), cuyos beneficios repercuten en todos los miembros de la sociedad y por los que no es viable que se pague de manera individual, en tanto quienes no pagaren obtendrían los mismos beneficios que los que sí lo hicieran (bienes públicos), deben ser asumidos por el Estado y no por las empresas de salud privadas, dedicadas a proveer los bienes privados. Esta concepción no tiene en cuenta que el carácter público de los bienes o de las acciones está determinado por el significado y el valor que la sociedad le otorga a dicho bien, si este constituye una prioridad social y si la sociedad decide financiarlo colectivamente y no porque su consumo sea individual (16). Además, divide de forma tajante las acciones colectivas de las individuales y fragmenta aún más la atención sanitaria, imposibilitando la articulación entre el campo de la asistencia en salud y la prevención y promoción de la salud. Esto se puede ver de forma clara en la reforma de la salud en Colombia, ${ }^{3}$ donde se registró una disminución muy importante de actividades orientadas a la prevención como la vacunación, cuyas coberturas descendieron de $90 \%$ en 1996, a $70 \%$ en 1999, para los niños menores de un año (18). Ello significó un aumento de epidemias de enfermedades como dengue, sarampión, polio, fiebre amarilla, etcétera. Fue notoria también la reducción en la prevención y diagnóstico temprano de la tuberculosis, que representa uno de los problemas más graves del país actualmente. Esta situación se presentó porque el Estado creía que la "competencia" estimula-

\footnotetext{
3 Dicha reforma se llevó a cabo en 1993 y organismos internacionales como el Banco Mundial la han calificado como un ejemplo a seguir para los países de la región latinoamericana, ya que ha observado los lineamientos de introducción de la libre competencia, la privatización, la descentralización, etc., en su sistema sanitario.
} 
ría el ejercicio de tales funciones por parte de las empresas aseguradoras y prestadoras de servicios, lo que en la práctica no quedó confirmado.

Por otro lado, los enfoques "tecnocráticos" concentrados en las transformaciones institucionales han dejado de lado el impacto que las políticas de ajuste estructural tuvieron sobre las condiciones de vida y de salud de la población en América Latina. Dichas políticas no solo no estimularon el crecimiento económico esperado, sino que incrementaron las ya históricas desigualdades sociales. Así, en 1990 había 48 millones de pobres en la región y para el año 2002 esta cifra había aumentado a 57 millones (19).

A pesar de que instituciones como el FMI y el BM han incluido la prevención y la promoción como parte de las estrategias sugeridas para lograr cambios significativos en la salud de la población latinoamericana, es importante anotar que, por un lado, mantienen la filosofía neoliberal de ubicar la salud en el ámbito de lo privado y considerarla bajo ciertas condiciones como tarea pública, orientando las políticas en salud a las prioridades de ajuste fiscal y, por otro lado, sus recomendaciones se limitan principalmente a la asistencia médica individual y no relacionan el proceso de cambios en el sector con estrategias que actúen sobre los determinantes sociales de la salud. Por el contrario, como se analizará más adelante, han fortalecido el componente de los estilos de vida que enfatiza los factores de riesgo individual y genera en muchos casos el efecto de "culpabilizar a la víctima".

\section{Las discusiones se han centrado en asuntos formales y no hay una reflexión de los modelos teóricos que sustentan los servicios de salud}

Como se señalaba en el anterior punto, las preguntas que han predominado en las reformas del sector salud han hecho referencia fundamentalmente a cómo ahorrar dinero, cómo hacer más eficiente la prestación sanitaria o cómo responder a las preferencias de los consumidores, las cuales son importantes en tanto se orientan a un mejoramiento de los aspectos organizativos de los sistemas de salud. No obstante, es necesario reflexionar sobre los problemas presentes en el modelo biomédico, que sigue siendo hegemónico en la práctica clínica, y sobre los "fines" y no solo los "medios" que están guiando las reestructuraciones sanitarias.

El paradigma biomédico plantea varios problemas que influyen de manera determinante en el ejercicio médico y en la formulación de las políticas de salud, entre ellos:

a) Fundamenta su conocimiento en el método experimental, estrechamente relacionado con una actitud positivista que considera únicamente válido lo "medible y cuantificable", expresado en leyes universales; por ello, se considera inválida cualquier explicación fuera de este esquema. Con esta concepción se consolida la comprensión mecanicista de la vida que considera a los seres humanos como partes desagregadas y fragmentadas, separadas entre sí pero ensambladas para ejecutar una función determinada. En el campo de las políticas públicas, la salud y la enfermedad se plantean como problemas exclusivamente individuales y no como fenómenos que hacen parte de un proceso centrado en las colectividades. No se tiene en cuenta que la historia de la comunidad, sus formas de organización, su comportamiento productivo, la religión, la filosofía, la educación (todo aquello que determina la actitud del hombre hacia la vida) condicionan el origen y desarrollo de las enfermedades y ejercen también influencia en la disposición individual a la enfermedad. Como lo expresa Montiel (20) citando a Canguilhem: "La salud no es solamente la vida en el silencio de los órganos, es también la vida en la discreción de las relaciones sociales".

b) La comprensión de la estructura física del ADN a mediados del siglo XX y los descubrimientos en torno al genoma humano, han revitalizado la concepción que sostiene que el problema de la enfermedad es principalmente una modificación de la biología del individuo, explicada en términos de las estructuras moleculares. No obstante, como lo afirma Golud (21), son muy pocas las enfermedades "monogénicas", es decir aquellas cuyo origen depende de un solo gen y en la mayor parte de ellas el entorno sociocultural, económico y político, juega un papel definitivo en su origen y desarrollo. De esta forma, a pesar de que una anomalía genética sea detectada, no existe la certeza de si el individuo enfermará o no; esta información tan solo indica que tiene mayor riesgo de enfermar (tiene la predisposición o "susceptibilidad") si se le compara con otra persona que sea genéticamente normal; incluso las personas que no tienen tal predisposición genética puede llegar a enfermar por factores relacionados con sus estilo de vida. Es decir que el contexto social interactúa con lo genético y lo biológico en el proceso de organización de las formas mismas de presentación y modificación de los procesos vitales del individuo (22).

Un elemento que se deriva de esta concepción del proceso de salud-enfermedad como un proceso social, es que compromete las políticas sociales, ya no únicamente en términos de necesidades de los servicios de salud, sino de las desigualdades sociales estructurales. La salud sería entonces la realización de un potencial biológico y cultural, la "capacidad de una persona de perseguir y realizar sus metas vitales y desenvolverse adecuadamente en un contexto social" (23).

c) El modelo biomédico fundamenta la concepción de la salud como un "estado", y ello se invalida cada vez más por los cambios ocurridos en el campo de la física atómica y cuántica, la teoría de la relatividad, los nuevos planteamientos de la ciencias de la complejidad y la teoría de los sistemas vivos auto-organizados las cuales, además de cuestionar conceptos básicos de la medicina occidental contemporánea, como la causalidad lineal, la tendencia a la comprensión mecanicista de la vida y la noción de cambio de corte neodarwinista, entre otras (24), han 
mostrado la necesidad de descentrar la salud de la especie humana y ubicar lo humano como una hebra más de la trama compleja de la vida, dando así un viraje del "antropocentrismo al biocentrismo". Según Maldonado (25), este nuevo enfoque intenta superar la ecología antropocéntrica que tiene al hombre como centro de todo valor, al hombre como separado de la naturaleza o por sobre ella y a la naturaleza como algo instrumental para hacer uso de ella. Así, el referente en el proceso de toma de decisiones en salud ya no serán solo los seres humanos, sino todas las demás formas de la vida distintas a las eminentemente humanas.

La reflexión de la forma como se conciben los fenómenos de la salud y la enfermedad en el modelo biomédico actual, pone en evidencia los problemas teóricos de este paradigma y la importancia de desarrollar trabajos interdisciplinarios que permitan comprender el proceso de salud- enfermedad como un proceso social. Dicha reflexión involucra la discusión con respecto a los modelos de educación médica y constituye un desafío que no se tuvo en cuenta en los diagnósticos que sustentaron las reformas sanitarias de los años noventa.

En un contexto de políticas de salud que fundamentalmente piensan en acciones orientadas a los individuos (sus formas de consumo de los servicios asistenciales, acciones preventivas en sus estilos de vida, etc.) y en la intervención institucional como eje del cambio sectorial, es necesario que se supere la concepción biomédica de la salud y que se puedan construir estrategias para que la población pueda ejercer control sobre los determinantes de su salud. De esta manera, los conceptos de "intersectorialidad" y de la política de salud como política social adquieren un nuevo significado, pues como lo explica Almeida (2): "no se trata de acoplar elementos de salud en otras políticas, sino de formularlas en el sentido que preserven las condiciones de vida y de salud de las poblaciones".

\section{La creencia de que el "acceso por sí solo" garantiza la equidad en salud}

Uno de los problemas más importantes en el desempeño de los sistemas de salud en la década de los noventa es la forma como se establece el equilibrio entre metas como la eficiencia y la equidad. Con el propósito de minimizar los costos y maximizar la provisión eficiente de los servicios, las reformas sanitarias de los años noventa en América Latina han defendido el establecimiento de un conjunto de parámetros administrativos conocidos con el nombre de "medicina gestionada", a través de los cuales se busca influir en las decisiones clínicas de los médicos por medio de incentivos financieros para que estos restrinjan al máximo las hospitalizaciones, las pruebas y los procedimientos, con el objeto de corregir el antiguo sistema de pago por servicio en el que los médicos se excedían en el uso de los recursos sanitarios. Los defensores de la "medicina administrada" arguyen que el paradigma de la medicina tradicional le ha dado mucho énfasis al buen manejo de lo clínico, dejando de lado los aspectos administrativos y financieros, los cuales también afectan el desempeño de la atención sanitaria (26). Ciertamente, las decisiones clínicas deben tener en cuenta la forma como los recursos de la sociedad se utilizan y administran; no obstante, es importante recalcar que estos niveles deben pensarse para mejorar la atención sanitaria y no para sacrificarla en aras de las ganancias económicas. Cabe reconocer que la búsqueda de la eficiencia puede ser un buen "medio" para el mejoramiento del control de costos y de la calidad de los servicios, pero el problema es que en las actuales reformas se ha convertido en un fin en sí mismo, que incluso está por encima de otros valores fundamentales como la equidad.

El principio de la equidad en la formulación de las reformas se ha enfocado principalmente a mejorar el acceso de los servicios de atención sanitaria, a través de políticas de focalización a los grupos poblacionales más necesitados. Uno de los problemas de esta estrategia es que, ante la evidente y dramática expansión de la pobreza en toda la región latinoamericana, dicha focalización no se hace en función de las necesidades básicas insatisfechas, sino a partir del techo presupuestal fijado por la política fiscal restrictiva y, por tanto, esta resulta ser rígida y siempre insuficiente. Las salidas habituales a los dilemas que esta situación presenta son varias: se aplican los recursos hasta donde alcanzan; se aplican criterios de doble o triple focalización (mujeres pobres, niños pobres, mujeres rurales pobres con hijos, etc.) o se aplica el criterio de costo-efectividad (17). Desde el punto de vista de los derechos sociales, se considera que el Estado debe dar respuesta a las demandas populares, pero los beneficios provistos son mirados más como "concesiones" que como "derechos". De esta manera, como lo señala Burkett (27), se presenta un desplazamiento del compromiso de la sociedad de garantizar los derechos sociales a través de las acciones públicas a los programas asistenciales y discrecionales para los pobres, la reducción del gasto social público, la definición de un paquete de servicios mínimos y el traslado de la mayor parte de los servicios sociales al mercado.

La racionalidad económica utilizada para seleccionar a los grupos de población beneficiarios de las ayudas del Estado y los cálculos costo-efectividad para medir la maximización de los recursos sanitarios en los programas sanitarios, ${ }^{4}$ definen el valor de las políticas de salud y de las intervenciones clínicas según el principio de la "generalización", por el cual se busca "el mayor bien para el mayor número", en oposición al principio de la "universalización" que plantea la búsqueda del "bien de todos y cada uno, por igual" (28). Desde este enfoque "utilitarista"de la justicia sanitaria se desestima el principio de la "igualdad de oportunidades", pues no se tiene en cuenta que las personas pueden generar diferencias de salud por motivos de los cuales no son responsables como la edad, el gé-

\footnotetext{
4 Los indicadores que han logrado mayor reconocimiento internacional son el de los años de vida ajustados con calidad, QALY, y el de los años de vida ajustados en función de la discapacidad: DALY.
} 
nero, la raza o las condiciones económicas, sociales y culturales, y sacrifica la equidad en aras de la eficiencia ya que siempre opera sobre la base de que lo importante es la maximización de los beneficios sanitarios globales y no los individuales; esto hace que se produzcan importantes inequidades en el acceso y uso de los servicios sanitarios. A lo anterior se agrega el hecho de que en la selección de los contenidos del paquete básico de salud se concede a los técnicos y políticos la última palabra sobre decisiones relacionadas con la vida y la calidad de vida de las poblaciones.

Además, en el contexto de introducción de los mecanismos del mercado en la atención sanitaria, se conciben los servicios de salud como "discrecionales" (a criterio o a gusto de cada uno), igualándolos con cualquier objeto de consumo o mercancía. Lo anterior resulta bastante problemático desde el punto de vista de la equidad, si se tiene en cuenta que las dificultades en el acceso a dichos servicios pueden significar la diferencia entre la vida o la muerte y las condiciones aceptables para el desarrollo de las capacidades humanas o daños biológicos o mentales irreversibles. En este mismo sentido, la consideración de la atención sanitaria como un "bien privado" que se compra en el mercado, está legitimando la mayor participación del sector privado en la provisión de servicios sanitarios; esto ha favorecido, según Armada y cols. (15) la transferencia de recursos (ingresos, riqueza, seguridad ambiental y poder político) de los países del Tercer Mundo a los más industrializados.

Por otro lado, en las reformas latinoamericanas poco se ha tenido en cuenta que la igualdad de acceso no implica igualdad de uso. Según Jensen y Money (29), la igualdad de acceso se consigue cuando se declara un derecho a consumir los recursos sanitarios, pero la igualdad de uso está determinada por múltiples factores como la información de los beneficios de la atención sanitaria, la localización geográfica, las barreras administrativas (tiempos de espera en las citas, horas de disponibilidad de los servicios, escasos tiempos de consulta, servicios accesibles en términos de distancia), de tipo financiero (copagos, cuotas moderadoras, el costo del transporte para acceder a los servicios de atención) o barreras culturales (nivel educativo, etnia, edad, género, diferentes percepciones de los individuos sobre su estado de salud, poca información disponible sobre los beneficios de la atención sanitaria, actitudes desfavorables de los proveedores hacia los pacientes, etc.) que dificultan a muchas personas el acceso real a los servicios de salud y que, además, escapan del control del individuo. El caso de Colombia lo ilustra muy bien, pues los proponentes de la reforma de salud del año 1993 consideraban que si se aumentaba el aseguramiento se eliminarían las barreras al acceso a los servicios, en especial las de tipo financiero que históricamente han caracterizado a este sistema de salud. Sin embargo, según Echeverri (30) la utilización efectiva de los servicios - acceso real- no se ha modificado sustancialmente: cerca de la mitad de la población de los estratos socioeconómicos más bajos en nueve ciudades colombianas no utilizan los servi- cios aduciendo, en $66 \%$ de los casos, razones económicas (por los copagos y cuotas moderadoras que deben hacer); los pacientes con enfermedades de alto costo (especialmente los del régimen subsidiado ${ }^{5}$ ) o que necesitan tratamientos de alta complejidad, relatan negación o trabas para recibir la atención. En otros casos, los aseguradores no fomentan la utilización de servicios con la exigencia de trámites, o con la ubicación de prestadores en zonas distantes para el usuario. Estas evidencias demuestran que el simple aseguramiento no garantiza el acceso real a los servicios.

En las reformas tampoco se ha tenido en cuenta que "la equidad en el consumo de los servicios de salud es condición importante, pero no suficiente para disminuir las desigualdades en el enfermar o morir existente entre los grupos sociales" (2). Las inequidades en salud son todas aquellas diferencias en las condiciones de salud que se originan en situaciones socioeconómicas, culturales o medioambientales que son innecesarias, evitables e injustas (31).

Esto no significa que las políticas orientadas a lograr la equidad eliminen todas las diferencias en la salud entre las personas, ya que las variaciones biológicas son inevitables; lo que se debe buscar es que reduzcan o eliminen las diferencias que pueden ser controladas en la estructura social. El problema está en la determinación de lo que es evitable, innecesario e injusto y las relaciones entre estas condiciones; por ello, como lo explica Ferrer (32), un desafío teórico en plena vigencia es: "Reflexionar sobre qué significa justicia en salud, cuáles son las fuentes de esa injusticia, cómo contribuye la justicia social a la justicia en salud, qué relaciones existen entre salud y desarrollo ..."

Las evidencias disponibles muestran la existencia de un mejoramiento de las condiciones de salud a medida que se asciende en la escala social. De esta manera, aquellos que estén privados del acceso a los servicios de atención hospitalaria y también quienes carezcan de los beneficios de las políticas para mejorar las condiciones de vida (nutrición, educación, vivienda, los trabajos bien remunerados, tiempo para el ocio, acceso a servicios de acueducto y alcantarillado, participación social, etc.) encuentran serias limitaciones en su "libertad de acción" para llevar el tipo de vida que quieren y valoran, en la posibilidad de recurrir a opciones reales entre las que pueden elegir, de reducir el sufrimiento y el padecimiento provocado por las enfermedades y de tener la oportunidad de prevenir la muerte "prematura".

En este sentido, en América Latina, a pesar de las proyecciones económicas y sociales que organismos financieros internacionales -Fondo Monetario Internacional, Banco Mundial, Banco Interamericano de Desarrollo- hicieron en los años noventa, las desi-

\footnotetext{
5 La reforma de salud en Colombia estableció tres regímenes: el subsidiado para la población más pobre y sin capacidad de pago, financiada por el Estado y por los trabajadores que cotizan; el contributivo, para la población trabajadora con capacidad de pago y su familia, y el régimen de los "vinculados" para la población no asegurada que paga una parte de su atención de su propio bolsillo y debe ser atendida en la red pública hospitalaria.
} 
gualdades sociales y de la pobreza, de acuerdo con las estimaciones de la Comisión Económica para América Latina y el Caribe (33), siguen siendo extremadamente altas. Para 2006, 36,5\% de los habitantes de la Región se encontraban en situación de pobreza, y otros 13,4\% estaban en extrema pobreza o indigencia. Así, el total de pobres alcanzó los 194 millones de personas, de los cuales 71 millones eran indigentes. Estudios recientes $(30,34,35)$ para el caso de Colombia muestran cómo a menor estrato socioeconómico, mayor mortalidad por enfermedades infecciosas y transmisibles (más del doble que en los estratos más altos); la tuberculosis es responsable de $15,3 \%$ de las muertes del estrato $1, y$ solo de $6,8 \%$ en el estrato 6 ; la diarrea lo es de $21,7 \%$ de la mortalidad del estrato 1 , mientras que en el estrato 6 es de 3,3\%. De igual forma, la población rural no solo tiene una calidad de vida más precaria, sino que vive menos; en 1997 la esperanza de vida era 1,8 años menor que la urbana, con especial vulnerabilidad en la población indígena y negra del país.

Todas estas cifras muestran la necesidad de diseñar medidas para que los grupos más desafortunados puedan lograr ganancias de salud, a partir del reconocimiento de que es necesario trascender los límites del individualismo biomédico y estudiar las fuerzas sociales e históricas que generan las desventajas en estas personas.

\section{El excesivo énfasis en la responsabilidad individual en el origen de las enfermedades}

Como consecuencia de los problemas en el financiamiento de los sistemas sanitarios y de la restricción de los gastos, en las reformas sanitarias se ha hecho un gran énfasis en la responsabilidad individual o en los "factores de riesgo individual" en el origen de las enfermedades, aspecto que coincide con los cambios en las nociones de "riesgo de salud" y de "aseguramiento" generados a partir de los nuevos y revolucionarios progresos en la ingeniería genética, la expansión de la teoría sobre los estilos de vida, así como también la intención de quitarle responsabilidades al Estado en el financiamiento y en la provisión de la atención sanitaria propuesta desde las políticas neoliberales.

La posibilidad de que a través de la medicina genética se evalúen con mayor precisión las predisposiciones genéticas de cada individuo a desarrollar una serie de enfermedades, cambia el sentido de la noción de riesgo y consolida un enfoque de aseguramiento más "individualista y determinista", que le concede un gran peso a los comportamientos personales de cada individuo y a los costos sociales de las actitudes individuales. Como lo expresa Rosanvallon (36):

ya no puede comprendérsela [la noción de riesgo] según un modo estadístico global, como cuando se calcula, por ejemplo, que cada individuo tiene una posibilidad $\mathrm{X}$ de morir de cáncer del hígado. Con el conocimiento genético, se pasa del "grupo" al "individuo" para personalizar el riesgo . . . así desde ahora es posible tender hacia el establecimiento de tablas individuales de riesgo, resultantes de la suma de lo innato y de lo adquirido.

Y aunque, en efecto, la "medicina predictiva" sigue haciendo los diagnósticos en términos de probabilidades, no por ello es menos cierto que introduce una ruptura, pues pone en tela de juicio el enfoque estadístico de lo social en beneficio de la elaboración de modelos que tienen más en cuenta los comportamientos personales. Esta transformación en la noción de aseguramiento conduce necesariamente a un cambio en la concepción de equidad en el campo sanitario, pues el concepto de justicia que sustentó la política de salud del Estado del Bienestar puso mayor énfasis en responsabilidad del conjunto de la sociedad en los problemas relacionados con las enfermedades y, por lo tanto, en la solidaridad como principio fundamental.

Al respecto, es importante anotar que es innegable que hoy, ante los problemas de racionalización de recursos, es fundamental que los individuos sean conscientes de su responsabilidad tanto en la prevención de enfermedades como en el uso adecuado de los recursos sanitarios, para lograr un sistema de salud que logre armonizar lo médicamente necesario con lo económicamente financiable. No obstante, a menudo resulta muy difícil establecer con certeza el papel de los diferentes factores causales (los individuales relacionados con la herencia y el estilo de vida y los de tipo social) en razón de la complejidad de las conexiones que dan origen a la enfermedad y por las limitaciones en los conocimientos de muchas enfermedades. En el caso del cáncer de pulmón, por ejemplo, es difícil determinar si es un problema solo del individuo o si se conjuga con otros factores como ser consumidor pasivo, la contaminación ambiental, las condiciones de trabajo, de la herencia, etcétera (37).

Por su parte, la teoría de los estilos de vida articula las relaciones existentes entre las elecciones y los hábitos individuales con el estatus de salud de las personas, partiendo del presupuesto ético de la libertad que tienen los seres humanos para definir su forma de vida y transformar el entorno en aras de lograr los ideales y los objetivos que desean. El problema de esta perspectiva es que se tiende a "culpabilizar a la víctima", pues no se tiene en cuenta - como lo plantean las teorías de justicia de los igualitaristas liberales- si existen y se disfrutan de manera real las libertades fundamentales para que los individuos tomen decisiones por sí mismos y asuman las responsabilidades que les corresponden. Al responsabilizar a los individuos de sus preferencias se ignora, como lo plantea Sen (38), la influencia que las desigualdades en los recursos y en las oportunidades tienen en la formación de dichas preferencias; así por ejemplo, muchas personas o grupos con bajo nivel de vida se empiezan a resignar con su situación y a concentrar sus preferencias en aquellas cosas que parece que pueden alcanzar, sin aspirar a aquellas que no podrían lograr ("preferencias adaptativas"). Igualmente, muchas personas pueden adquirir enfermedades por el desconocimiento de los ries- 
gos en el momento de exponerse a ellos, por lo cual sería injusto que el individuo que sufre la enfermedad sea considerado responsable de ella.

Además, la insistencia en que las enfermedades son problemas de los trabajadores "poco responsables" o de ciudadanos que "no se cuidan", establece un complejo código de premios y castigos en función de las capacidades del individuo para conservar su salud que evita la crítica del statu quo y la reflexión de los factores socioeconómicos y políticos que intervienen en la etiología de las enfermedades (39). Con este enfoque, como lo expresa Vicente Navarro (40), se fomenta el individualismo como valor en los sistemas sanitarios y se reafirma "la individualización de una causalidad colectiva que, por su misma naturaleza, hubiese requerido una respuesta colectiva".

Por otro lado, el énfasis en la responsabilidad individual en el origen de las enfermedades está fuertemente influido por la emergencia del modelo neoliberal que busca reemplazar la noción de "universalidad" — que presupone un compromiso solidario de la sociedad para garantizar con medios públicos el acceso de todos los ciudadanos a los servicios de atención sanitaria-, por la idea de la "selectividad", aplicada a los programas asistenciales y discrecionales para los más pobres. Del mismo modo, se intenta desplazar el concepto de justicia distributiva que compromete directamente al Estado y generalizar el concepto de "justicia conmutativa", según el cual cada individuo recibe de acuerdo al equivalente a sus aportes o contribuciones. Desde esta perspectiva, la definición de la atención sanitaria como un "bien social"en el cual la provisión de servicios a cada individuo es valiosa para la sociedad en su conjunto, es cambiada por una concepción que la define como un "bien privado", es decir, como una mercancía que es consumida por cada individuo en cantidades variables y adquirida según lo que las personas deseen y puedan comprar en el mercado. En este nuevo escenario se busca que cada uno se responsabilice del hecho de enfermar.

Como ya se había señalado en las políticas de prevención y promoción que se esbozan en las reformas sanitarias de la década de 1990, existe una fuerte tendencia a que predomine el enfoque de la "responsabilidad individual"; por ello, se busca que los esfuerzos educativos en salud estimulen los comportamientos que reduzcan el riesgo y mejoren la salud de los individuos. Dichos esfuerzos son de indudable importancia, siempre y cuando se recurra a políticas intersectoriales que asuman las causas sociales de la enfermedad e intervengan sobre ellas. Es fundamental evaluar las barreras que los ciudadanos pueden tener para acceder a los programas de prevención y promoción y la consideración de las personas no como simples vehículos pasivos de las políticas, sino como interlocutores válidos en todo el proceso de toma de decisiones en salud. Esta perspectiva coincide con los planteamientos que buscan ir más allá de la gestión episódica de la enfermedad, consultando las necesidades y los problemas de salud de los individuos, la familia y la comunidad; fomentar el trabajo multidisciplinario, de modo que los distintos profesionales de la atención primaria en salud trabajen juntos para lograr una asistencia coordinada o "sin fronteras" entre los servicios clínicos especializados, de bienestar social y de salud pública; una función educativa para los cuidadores, pacientes y profesionales (41).

\section{CONCLUSIONES}

La reflexión sobre los criterios de las reformas sanitarias de los años noventa en América Latina, que definieron su contenido y sus orientaciones, muestra la necesidad de que en los procesos de formulación de las políticas del sector de la salud se tengan en cuenta aspectos como: a) el reconocimiento de que el acceso a los servicios de atención sanitaria es solo un factor que incide en las condiciones de salud de la población, y es por ello que no se pueden dejar de lado los procesos que subyacen a la mala salud de las personas, contemplando los múltiples determinantes de la salud; b) la necesidad de superar la concepción biomédica de la salud, desarrollando enfoques interdisciplinarios y planteando estrategias de trabajo "intersectorial"; c) la necesidad de buscar el acceso real a los servicios de atención sanitaria a través de un trato equitativo con los que tienen la misma necesidad sanitaria (equidad horizontal) y de dar preferencia a aquellos con mayores necesidades (equidad vertical). Es imprescindible tener en cuenta que la equidad en el consumo de los servicios de salud es una condición importante pero no suficiente para lograr la equidad en salud; d) si bien los factores relacionados con los estilos de vida son elementos importantes para prevenir o mitigar los efectos de ciertas enfermedades, la evaluación de las desigualdades en los recursos y en las oportunidades que las personas tienen en la elección de sus hábitos individuales es necesaria.

De la misma forma, la incorporación de la competencia y los mecanismos del mercado en la mayoría de las reformas sanitarias hace necesario traer a la mesa de discusión la cuestión de si el principio de justicia distributiva en el ámbito de la sanidad debe ser diferente al que rige el mercado pues, como lo plantea Walzer (42), los principios de justicia dependen del significado social que se le atribuya a cada bien social y del reconocimiento de que no hay un principio "único" de justicia que gobierne todos los bienes sociales, sino que existen diferentes "esferas de la justicia".

\section{SYNOPSIS}

\section{Deficiencies in evaluating health care reforms in the 1990s in Latin America}

Some of the recurring themes seen on health-sector reform agendas of Western countries (those at the center as well as those on the periphery) over the last decade have been: the debate over new ways of organizing health systems, with sights set on achieving greater efficiency and quality; redefining health care benefit packages and services to better 
distribute health resources; and incorporating market mechanisms into the health care environment to better respond to expectations of health care consumers.

The fundamental purpose of this article is to analyze certain concepts that define and explain the origin of the 1990s health care reforms in Latin America and to refute some of the more important principles, such as: the belief that improving the functional efficiency of the health care system alone would improve the health of the population; the excessive concern with the administrative and structural aspects of health sys- tems without a discussion of the underlying theoretical models; the idea that access, in and of itself, can guarantee "equity in health"; and lastly, the undo emphasis that was placed on individual "risk factors" as the cause of all illness.

Key words: health systems; public health; health policy, planning, and management; equity in health; Latin America.

\section{REFERENCIAS}

1. Almeida CM. Os modelos de reforma sanitária dos anos 80: uma análise crítica. Revista Saúde e Sociedade. 1996; 5(2):3-57

2. Almeida CM. Reforma del sector salud y equidad en América Latina y el Caribe: conceptos, agenda, modelos y algunos resultados de implementación. Gerencia y Políticas de Salud. 2005;4(9):6-60.

3. Saltman R, Figueras J. Reforma sanitaria en Europa. Análisis de las estrategias actuales. Madrid: OMS, Ministerio de Sanidad y Consumo; 1997.

4. McKeown T. El crecimiento moderno de la población. Barcelona: Antoni Bosch Editor; 1978.

5. McKeown T. El papel de la medicina. ¿Sueño, espejismo o némesis? México: FCE; 1989.

6. Terris M. La revolución epidemiológica y la medicina social. Mexico: Siglo Veintiuno Editores; 1987.

7. Lalonde M. A new perspective on the health of Canadians: a working document. Ottawa: Information Canada; 1975

8. Blum J. Planning of health. Berkeley: University of California Press; 1979.

9. OMS. Informe sobre la salud en el mundo 2000. Mejorar el desempeño de los sistemas de salud. Ginebra: OMS; 2000.

10. Colomer C, Alvarez-Dardet C. Promoción de la salud y cambio social. Barcelona: Masson; 2001.

11. Evans RG, Barer ML, Marmor TR. Why are some people healthy and others not? The determinants of health of populations. New York: Aldine de Gruyter; 1994.

12. Gamboa T, Arellano M. Tendencias neoliberales en la reforma de la gestión pública en América Latina. Gerencia y Políticas de Salud. 2007;6(13):46-65.

13. Granados R. La reforma de los sistemas de salud: tendencias mundiales y efectos en Latinoamérica y el Caribe. Gerencia y Políticas de Salud. 2002;1(3):16-46.

14. Almeida CM. Reforma de sistemas de servicios de salud y equidad en América Latina y el Caribe: algunas lecciones de los años 80 y 90. Cad Saude Publica. 2002;18(4):905-25.

15. Armada F, Muntaner C, Navarro V. Health and social security reforms in Latin America: The convergence of the World Health Organization, the World Bank, and transnational corporations. Int J Health Serv. 2001;31(4):729-68.

16. Laurell A, López O. Market commodities and poor relief: the World Bank pro- posal for health. Int J Health Serv. 1996; 26(1):1-18.

17. Laurrell A. La política de salud en el contexto de las políticas sociales. Gaceta. 2000;1(1):44-54

18. Ahumada C. La penuria de la salud pública. Gerencia y Políticas de Salud. 2002;1(3):47-57.

19. Human Development Report. Millennium Development Goals: A compact among nations to end human poverty. UNDP. Oxford University Press. New Cork. 2003. [Sitio en Internet] Hallado en: http://hdr.undp.org/en/media/ hdr03_complete.pdf. Acceso el 15 septiembre 2007.

20. Montiel L. "Beneficios y riesgos del nuevo valor de la salud en la sociedad pos-industrial". En: Arquiola E, ed. La salud en el Estado del Bienestar. Cuadernos Complutenses de Historia de la Medicina y de la Ciencia No. 2 . Madrid: Universidad Complutense de Madrid; 1992. P. 46.

21. Golud E. Los límites de la medicina. Santiago de Chile: Editorial Andrés Bello; 1996.

22. Quevedo E. El proceso de salud-enfermedad: hacia una clínica y una epidemiología no positivista. En: Cardona G, ed. Sociedad y salud. Bogotá: Zeus Editores; 1992. Pp. 7-44.

23. Callahan D, Allert $G$, Boyd $K$, Gillon $R$, Glasa J, Nordenfelt L, et al. Los fines de la medicina. El establecimiento de nuevas prioridades. Proyecto Internacional del Hastings Center. Cuadernos de la Fundación Víctor Grífol i Lucas No. 11. Barcelona: Fundación Víctor Grífol i Lucas; 2004.

24. Cárdenas H. Medicina comunitaria y medicina tradicional indígena. En: $\mathrm{Zu}$ luaga $G$, Correal C, comps. Seminario Internacional de Etnomedicina. Bogotá: Universidad El Bosque; 2002. Pp. 194-9.

25 Maldonado C. Filosofía y salud. En: Escobar J, Jácome S, Cardona G, Málaga $\mathrm{H}$ Rodríguez C, Mont J, et al. Bioética y calidad de vida. Colección Bios y Ethos No. 9. Bogotá: Ediciones El Bosque; 1997. Pp. 65-80.

26. Agich G. The importance of management for understanding managed care. J Med Philos. 1999;24(5):518-34.

27. Burkett P. Poverty crisis in the Third World: the contradictions of World Bank Policy. Int J Health Serv. 1991;21:471-80.

28. Gracia D. Ética de la eficiencia. En: Amor J, Ferrando I, Ruiz J, eds. Ética y gestión sanitaria. Madrid: Universidad Pontificia Comillas; 2000. Pp 43-54.

29. Jensen $U$, Mooney G. "Changing values and changing policy". En: Jensen $U$, Mooney $\mathrm{G}$, eds. Changing values in medical and health care decision making. Chichester: John Wiley \& Son; 1990. Pp. 179-89.

30. Echeverri E. La salud en Colombia: abriendo el siglo ... y la brecha de las inequidades. Gerencia y Políticas de Salud. 2002;1(3):76-93.

31. Whitehead M. The concepts and principles of equity and health. Int J Health Serv. 1992;22(3):429-45.

32. Ferrer Lues M. Equidad y justicia en salud. Implicaciones para la bioética. Acta bioeth. [Publicación periódica en línea]. 2003. [citada 2007 Agos 8]; 9 (1): [13 pantallas]. Hallado en: URL: http:// www.scielo.cl/scielo.php?script=sci_art text\&pid=S1726-569X2003000100011\& $\operatorname{lng}=\mathrm{es} \& \mathrm{nrm}=$ iso

33. Cepal. Panorama social de América Latina 2007. [Sitio en Internet] Hallado en: http://www.eclac.org/id.asp?id= 30305. Acceso el 14 abril 2008.

34. Yepes L. La salud en Colombia. Estudio sectorial de salud. Bogotá: Ministerio de Salud. Departamento Nacional de Población; 1990.

35. Málaga H, Latorre M, Cárdenas J, Montiel $\mathrm{H}$, Sampson $\mathrm{C}$, Taborda MC, et al. Equidad y reforma a la salud en Colombia. Rev Salud Publica (Bogota). 2000; 2(3):193-219.

36. Rosanvallon P. La nueva cuestión social. Repensar el Estado Providencia. Buenos Aires: Manantial; 1995.

37. Beachamps T, Childress J. Principios de ética biomédica. Madrid: Masson; 1999.

38. Sen A. Bienestar, justicia y mercado. Barcelona: Paidos; 1997.

39. Crawford R. Usted es un peligro para su salud: aspectos ideológicos y políticos de la práctica de culpabilizar a la víctima. En: De la Cuesta C, comp. Salud y enfermedad. Lecturas básicas en sociología de la medicina. . $^{\mathrm{a}}$ ed. Medellín: Universidad de Antioquia; 1999. Pp. 47-76.

40. Navarro V. La medicina bajo el capitalismo. Barcelona: Crítica; 1978.

41. Mullan F. Community-oriented primary health care: an agenda for the $80 \mathrm{~s}$. N Engl J Med.1982;307:1076-8.

42. Walzer M. Las esferas de la justicia. Una defensa del pluralismo y de la igualdad. México: FCE; 1997. 